\title{
Intoxicación de equinos por Senecio $p p$ en el noroeste argentino
}

\author{
Micheloud, J.F.,2; Merep, P.3; Tomas, R.H.3; Perotti, M.'3 Schuff, C³. \\ ${ }^{1}$ Área de Sanidad Animal -IIACSa CIAP/INTA, Salta. ${ }^{2}$ Facultad de Ciencias Agrarias y Veterinarias, \\ Univ. Cat. Salta (UCASAL), Argentina. E-mail: micheloud.juan@inta.gob.ar.
}

\begin{abstract}
Resumen
Micheloud, J.F.; Merep, P.; Tomas, R.H.; Perotti, M.; Schuff, C.: Intoxicación de equinos por Senecio sp en el noroeste argentino. Rev. vet. 28: 2, 126-131, 2017. La toxicidad de varias especies del género Senecio es bien conocida a nivel mundial. En Argentina se han descrito casos en bovinos pero los registros son escasos. En este trabajo se describen cuadros de seneciosis equina en dos predios rurales del noroeste argentino (Salta). Se mencionan los hallazgos clínicos y patológicos, caracterizados por fibrosis hepática, megalocitosis, hiperplasia biliar y colestasis. En individuos afectados se observó pérdida de peso, depresión, ataxia y dificultad para deambular. En las áreas de pastoreo se identificó la presencia de $\mathrm{Se}$ necio rudbeckiaefolius, S. madagascariensis y S. hieronymi. Se identificaron y cuantificaron alcaloides pirrolizidínicos en estas tres especies, mediante cromatografía gaseosa acoplada a espectrometría de masa. Se determinó la presencia de senecionina, integerrimina, usaramina, jacozina, jacobina, senecivernina, platyphyllina y neoplatyphyllina. Los resultados obtenidos confirman la intoxicación de equinos por Senecio en Argentina y ponen de manifiesto los primeros hallazgos de alcaloides pirrolizidínicos en tres especies de Senecio endémicas para el noroeste del país.
\end{abstract}

Palabras claves: equino, intoxicación, Senecio, alcaloides pirrolizidínicos, hepatotoxicidad.

\begin{abstract}
Micheloud, J.F.; Merep, P.; Tomas, R.H.; Perotti, M.; Schuff, C.: Senecio intoxication of equines from Argentinean northwest. Rev. vet. 28: 2, 126-131, 2017. The toxicity of several species of genus Senecio is well known worldwide. In Argentina there have been cases in cattle but records are scarce. In this paper equine seneciosis is described in two rural areas of argentinean northwest. Clinical and pathological findings characterized by hepatic fibrosis, megalocytosis, cholestasis and biliary hyperplasia, were recorded. In sick animals it was observed weight loss, depression, and ataxia. In grassing areas Senecio rudbeckiaefolius, Senecio madagascariensis and Senecio hieronymi were identified. Pyrrolizidine alkaloids were identified by gas chromatography coupled to mass spectrometry. Presence of senecionine, integerrimine, usaramine, jacozine, jacobine, senecivernine, platyphylline and neoplatyphylline was recorded. The results confirm Senecio poisoning in horses in Argentina and reveal the first finding of pyrrolizidine alkaloids in three endemic species of Senecio for Northwest Argentina.
\end{abstract}

Key Word: equine, intoxication, Senecio, pyrrolizidine alkaloids, hepatotoxicity.

\section{INTRODUCCIÓN}

Un gran número de etiologías se vinculan a trastornos hepáticos en los equinos, siendo los alcaloides pirrolizidínicos (APs) la causa tóxica más frecuentemente descripta. Entre las plantas más conocidas por su toxicidad que contienen estos alcaloides se pueden citar: Senecio sp (Asteraceae), Echium sp, Amsinckia $s p$, Cynoglossum officinale (Boraginaceae) y Crotalaria $s p$ (Fabaceae) ${ }^{25}$. Estas sustancias son fitoquímicos de ocurrencia natural, presente en unas 6000 especies de plantas de diversos géneros y familias ${ }^{8}$.

Recibido: 23 setiembre 2016 / Aceptado: 17 abril 2017
La intoxicación por Senecio es reconocida en todo el mundo, siendo bovinos y equinos las especies más frecuentemente afectadas ${ }^{9}$. Este género está integrado por más de 1200 especies distribuidas mundialmente. En Argentina se reconocieron 270 especies y 43 variedades, citándose 57 especies en la Provincia de Salta ${ }^{13}$.

La forma de presentación más habitual de la intoxicación por Senecio es crónica, y los signos clínicos ocurren semanas o meses después del consumo de la planta ${ }^{27}$. En nuestro país, las especies vinculadas a cuadros de intoxicación en bovinos son Senecio tweediei, S. pampeanus, S. grisebachii, S. selloiy S. madagascariensis ${ }^{18}$, pero no se identifican registros bibliográficos de intoxicación en caballos. 
El presente trabajo describe la intoxicación por $\mathrm{Se}$ necio en equinos, en dos regiones de la Provincia de Salta.

\section{MATERIAL Y MÉTODOS}

El estudio se realizó en dos localidades de la Provincia de Salta. El primer brote (foco 1) ocurrió en un establecimiento ganadero localizado en el paraje El Gallinato, Departamento La Caldera. El segundo (foco 2 ) afectó a un grupo de productores de la localidad de Potrero de Díaz, Departamento Chicoana. Ambos sitios corresponden a la eco-región chaco serrano, que se encuentra a unos 1.500 metros de altura sobre el nivel del mar y se caracteriza por valles y sierras donde el régimen pluviométrico es de 800 a $1200 \mathrm{~mm}$ anuales ${ }^{4}$

En ambos casos los animales eran mantenidos sobre pasturas naturales y eventualmente suplementados con grano. El motivo de consulta fue la mortandad de caballos "por goteo", con signos de decaimiento y pérdida de peso. A requerimiento de los productores, el Servicio de Diagnóstico Veterinario Especializado de INTA-SALTA efectuó visitas a estos establecimientos, identificándose dos animales afectados que fueron inspeccionados clínicamente.

En el foco 1, el establecimiento contaba con una tropilla de unos 20 caballos mestizos (criollo $\mathrm{x}$ árabe) y el animal afectado correspondía a una yegua de 15 años que se encontraba amamantando un potrillo de 4 meses de edad. En el foco 2, el animal era un macho castrado de unos 10 años de edad. De ambos animales se colectaron muestras de sangre para efectuar estudios hematológicos y bioquímicos.

Tras la muerte de los animales se les efectuó necropsia completa y se colectaron muestras de tejidos en formol bufferado al $10 \%$, las cuales se procesaron por técnicas histopatológicas clásicas de inclusión en parafina, seccionadas mediante microtomía y teñidas con hematoxilina-eosina. Además, para el caso del tejido hepático, se empleó la tinción tricrómica de Masson para evaluar la proliferación de tejido conectivo. Los potreros de pastoreo de los animales afectados fueron inspeccionados para buscar especies tóxicas y cuantificar su presencia.

Se recolectaron ejemplares vegetales que fueron herborizados para su identificación botánica. Estas muestras fueron registradas y archivadas en el Herbario MCNS de la Facultad de Ciencias Naturales de la Universidad Nacional de Salta. Los APs fueron extraídos mediante la técnica reportada por Benn \& $\mathrm{Gul}^{3}$.

La composición de los extractos de alcaloides fue determinada mediante un cromatógrafo gaseoso (Hewlett-Packard modelo 6890) con detector MS cuadrupolar HP 5973. La identificación de los compuestos se realizó por comparación de los espectros de masa obtenidos con los de la biblioteca de espectros del equipo: NBS75K, NIST (National Institute of Standards and Technology),
Wiley 275 y por comparación con los espectros reportados en la literatura ${ }^{28}$.

\section{RESULTADOS}

Hallazgos epidemiológicos. En ambos brotes existían antecedentes históricos de animales afectados por este cuadro, que se repiten año a año. El establecimiento correspondiente al foco 1 registraba pérdidas del 20 al $25 \%$ de los animales. Para el grupo de productores del foco 2, las pérdidas no pudieron ser cuantificadas, pero se mencionó que año tras año registraba la muerte de entre 8 y 10 caballos. En ambos casos, los decesos ocurrieron de septiembre a marzo y todos los individuos que enfermaron murieron, pese a los distintos tipos de tratamientos empleados por los propietarios.

Hallazgos clínicos. En los animales afectados, los signos clínicos se iniciaron bruscamente, aunque el cuadro fue progresivo, con períodos de mejora transitoria. En los casos observados, la afección se caracterizó inicialmente por pérdida de peso, debilidad, depresión y somnolencia; los animales revelaron un andar vacilante e incoordinación leve (más marcada a nivel de los miembros posteriores). Durante la permanencia en estación se mantenían con los miembros separados y la cabeza baja (como dormitando)

Uno de los individuos inspeccionados permaneció con los miembros anteriores cruzados. Eventualmente se observaron cortos períodos de alerta. Un signo que se registró con frecuencia fue que los individuos afectados, una vez aprehendido el alimento, lo mantenían en la boca sin deglutirlo. La evolución en el foco 1 fue mayor de 21 días y de 1 a 15 días en el caso del foco 2 . Los resultados de los parámetros bioquímicos del suero de ambos animales se exponen en la Tabla 1.

Hallazgos patológicos. Macroscópicamente las necropsias de los animales de ambos focos evidenciaron atrofia serosa de la grasa peri-renal y mesentérica, así como leve edema del meso-colon. En ambos casos, el hígado se encontraba reducido de tamaño y firme al tacto, con sus bordes afilados y al corte se observaba

Tabla 1. Hallazgos bioquímicos en los dos animales intoxicados (1 y 2).

\begin{tabular}{lcccccc}
\hline determinaciones & \multicolumn{2}{c}{ día 0} & \multicolumn{2}{c}{ día 7} & \multicolumn{2}{c}{ día 15} \\
\hline animal & 1 & 2 & 1 & 2 & 1 & 2 \\
BRR-T (mg/dl) & - & 0,5 & 0,9 & 1,0 & 1,6 & - \\
BRR-D (mg/dl) & - & 0,2 & 0,2 & 0,3 & 0,2 & - \\
GOT (U/l) & 174 & 230 & 604 & 1500 & 1349 & - \\
GGT (U/l) & - & 320 & 343 & 580 & 442 & - \\
ALP (U/l) & 788 & 780 & 411 & 820 & 355 & - \\
PT (g/dl) & 7,4 & 7,2 & 7,2 & 7,8 & 7,5 & - \\
ALB (g/dl) & 2,5 & 2,8 & 2,4 & 2,1 & 2,2 & - \\
\hline
\end{tabular}

BRR-T: bilirrubina total, BRR-D: bilirrubina directa, GOT: glutámico-oxalacético transaminasa, GGT: gamma glutamil transferasa, ALP: fosfatasa alcalina, PT: proteínas totales, ALB: albúmina. 


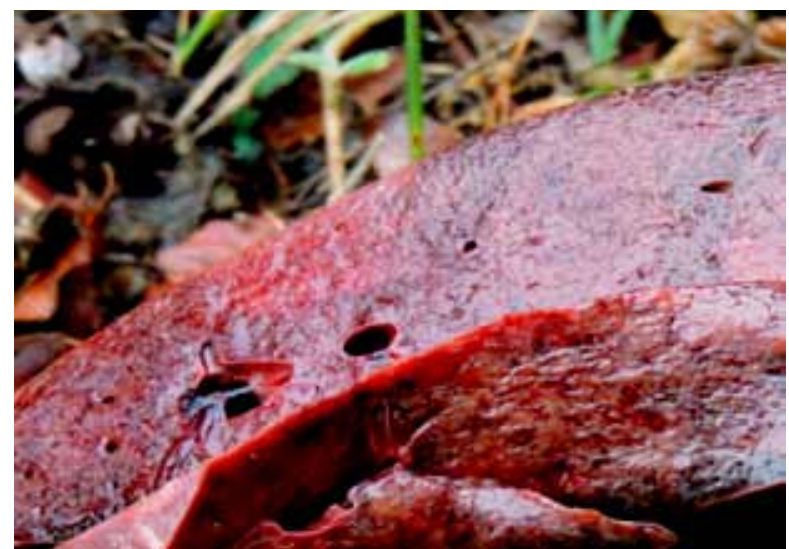

Figura 1. Al corte, el hígado reveló una acentuación del patrón lobulillar del parénquima.

una acentuación del patrón lobulillar del parénquima, con zonas pálidas intercaladas con áreas de intensa congestión (Figura 1).

A nivel del sistema nervioso central se apreció congestión meníngea. Los demás órganos no presentaron lesiones macroscópicas de relevancia. Microscópicamente se observó intensa fibrosis peri-portal, hiperplasia de los conductillos biliares y megalocitosis, caracterizada por formas celulares anómalas con márgenes irregulares y una apariencia difusa del citoplasma celular (Figura 2). En el parénquima hepático, las lesiones fibróticas difusas se evidenciaron mediante la tinción Tricrómica de Masson, que permitió visualizar la abundancia de fibras colágenas tipo I (Figura 3). Estos hepatocitos presentaban intensa marcación de núcleos $\mathrm{y}$ nucléolos. En algunos casos se observaron células con más de un núcleo.

Asociada a estas lesiones se pudo identificar la presencia de algunas células inflamatorias mononucleares (linfocitos y macrófagos) entre los hepatocitos. También pudieron apreciarse algunas zonas discretas de necrosis. En el caso del foco 1, la revisión histológica del tejido pulmonar reveló una neumonía intersticial con presencia de membranas hialinas y re-epitelización alveolar por neumocitos tipo 2. En el tejido nervioso se observó vacuolización de la sustancia blanca y astrocitosis Alzheimer tipo II.

Hallazgos botánicos. En el paraje El Gallinato (foco 1), al recorrer el potrero donde se alojaban los animales, fueron identificadas Senecio rudbeckiaefolius Meyen \& Walp (SR), S. madagascariensis Poir (SM) y $S$. hieronymi Griseb (SH), la especie más abundante en el área de pastoreo. En Potrero de Díaz (foco 2), se identificaron $S$. madagascariensis y $S$. rudbeckiaefolius en similares proporciones.

Hallazgos toxicológicos. En muestras procedentes de El Gallinato, los alcaloides identificados en SR fueron: senecionina $(92,6 \%)$, integerrimina $(5,8 \%)$, usaramina $(0,7 \%)$, trazas de senecivernina, platyphyllina y neoplatyphyllina $(<0,05 \%)$; jacozina $(50,4 \%)$, usaramina $(36,0 \%)$, senecionina $(6,2 \%)$, jacobina $(4,2 \%) \mathrm{y}$

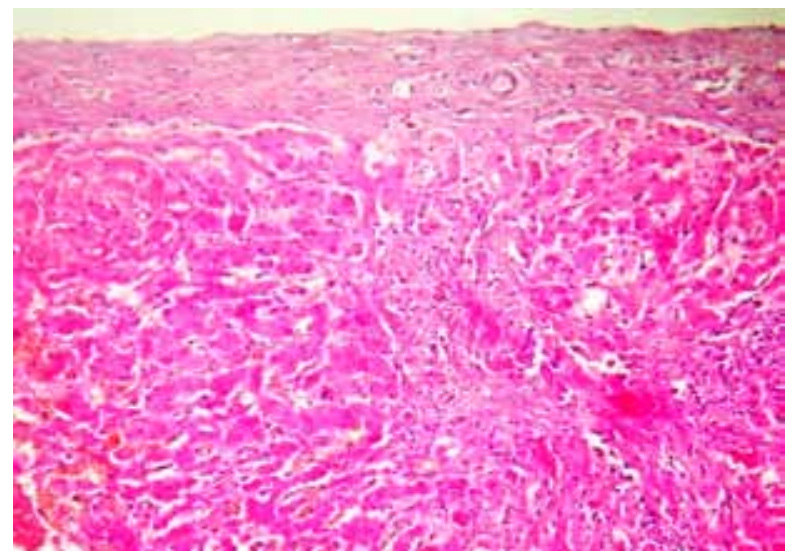

Figura 2. Fibrosis periportal con hiperplasia de conductillos biliares y megalocitosis (HyE).

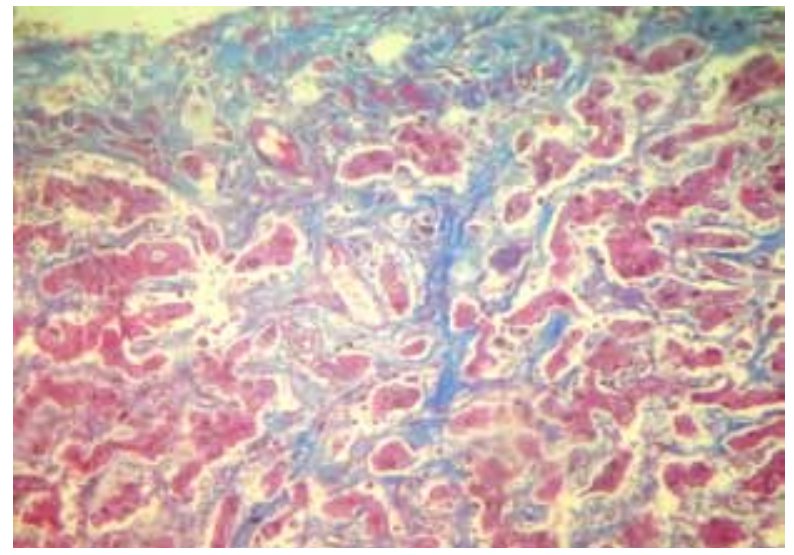

Figura 3. Fibrosis con abundantes fibras colágenas (azules) entre los hepatocitos (Tricrómica de Masson).

trazas de integerrimina en SH. Por su parte, en SM se reveló la presencia de senecionina $(66,1 \%)$, integerrimina $(9,7 \%)$, senecivernina $(2,3 \%)$, usaramina $(1,7 \%)$, platyphyllina $(0,9 \%)$ y trazas de neoplatyphyllina. No fueron detectadas diferencias cuali-cuantitativas significativas de estas muestras con relación a las recolectadas en Potrero de Díaz.

\section{DISCUSIÓN}

En la literatura puede encontrarse una serie de enfermedades de naturaleza endémica en equinos, causadas por el consumo de diversas especies de Senecio. La intoxicación con $S$. jacobea fue vinculado a stomach staggers en Gales (Gran Bretaña) ${ }^{25}$ y a winton disease en Nueva Zelanda ${ }^{17}$. Por su parte, S. burchelli, S. retrorsus y $S$. isatideus fueron relacionados a la enfermedad de dunziekte en Sudáfrica ${ }^{14}$. En Nebraska, $S$. riddellii es el causante de walking disease y S. erraticus -en el sur de Bohemia- sería el responsable de zdar disease $^{23}$

En Brasil se describen intoxicaciones en equinos atribuidas a $S$. brasillensisy $S$. oxiphyllus ${ }^{12}$; en Uruguay vinculadas con $S$. grisebachii ${ }^{21}$ y en Chile con $S$. erraticus ${ }^{1}$. En Argentina nuestro grupo no ha encontrado registros de intoxicación en caballos, mientras que las especies vinculadas a cuadros de intoxicación 
en bovinos son $S$. tweediei, S. pampeanus, S. grisebachii, $S$. selloi y $S$. madagascariensis ${ }^{18}$.

En cuanto a las especies estudiadas en este trabajo, la presencia de S. madagascariensis Poir, se extiende ampliamente en el país, abarcando Buenos Aires, Córdoba, Corrientes, Entre Ríos, Jujuy, La Pampa, Mendoza, Santiago del Estero, Santa Fe y Salta ${ }^{13}$. Por su parte, S. rudbeckiaefolius Meyen \& Walp, nativa de Bolivia, Perú y Argentina, crece en las provincias de La Rioja, Catamarca, Salta, Jujuy, San Luis y Tucumán ${ }^{5,13}$. S . hieronymi Griseb, es nativa de la región noroeste, extendiéndose hasta Mendoza y Córdoba, vegetando en los lugares húmedos de las selvas subtropicales, aunque puede llegar a los prados de $3500 \mathrm{msnm}^{5}$. Se afirma que la toxicidad de los senecios está determinada en gran medida, por la dosis total de APs ingeridos; dichos efectos son acumulativos y tienden a ser progresivos ${ }^{16}$.

El cuadro clínico en los equinos puede iniciarse a los 6-12 meses después de haber cesado la ingestión de estas especies tóxicas ${ }^{6}$, mientras que la evolución experimental varía de 1 a 30 días ${ }^{20}$. Por su parte, sintegerrimina y retrorsina fueron identificadas en S. brasiliensis, especie que experimentalmente resultó tóxica a dosis única de $8,5 \mathrm{~g} / \mathrm{kg}$ y $15 \mathrm{~g} / \mathrm{kg}$ y dosis repetidas de entre 1,74 a $9,66 \%$ del peso vivo ${ }^{20}$. De $S$. hieronymi y $S$. rudbeckiaefolius no existen antecedentes respecto a su toxicidad; en esta última especie, integrantes de nuestro grupo de trabajo (Merep et al. 2009) detectaron integerrimina y senecionina.

Es probable que las tres especies presentes en las áreas de pastoreo hayan contribuido a la aparición de los cuadros clínicos detectados en los establecimientos inspeccionados. Los signos nerviosos y la pérdida de peso constituyen el cuadro más frecuente advertido en equinos cuando se han intoxicado por Senecio ${ }^{11}$, lo cual es coincidente con lo observado en este trabajo.

El tiempo de exposición a la pastura infestada por Senecio puede ser variable, mencionándose que en casos espontáneos demandaría entre 3 a 60 días ${ }^{12}$. Por otra parte, la fibrosis hepática y la hiperplasia de conductillos biliares asociada a megalocitosis encontrada en estas localidades de la Provincia de Salta, es un hallazgo bastante sugestivo -aunque no exclusivo- de la intoxicación por alcaloides pirrolizidínicos ${ }^{25}$. Los APs bloquean la mitosis celular, dando lugar a la formación de megalocitos ${ }^{24}$.

El "estatus espongiosus" de la sustancia blanca en el sistema nervioso central y la astrocitosis Alzheimer tipo II resultan de la hiperamoniemia, producto de la insuficiencia hepática ${ }^{26}$, hallazgo que se observó en caballos intoxicados natural y experimentalmente con Senecio ${ }^{12,20,21}$.

Los estudios experimentales mostraron hiperactividad enzimática significativa en las fases tempranas del daño hepático, que luego tiende a declinar a rangos normales persistiendo así hasta los estadios finales, durante los cuales repentinamente vuelven a incrementarse de forma marcada ${ }^{6}$. Como las lesiones están en gran medida en la región portal, la gamma glutamil transfe- rasa (GGT), la fosfatasa alcalina (ALP) y la glutámico oxalacético transaminasa (GOT) tienden elevarse en el suero sanguíneo ${ }^{19}$.

En el presente caso la bioquímica sérica arrojó valores elevados de las tres enzimas en todas las ocasiones en las que se muestrearon los animales. Se afirma que las enzimas GGT y ALP mantendrían elevaciones moderadas durante todo el curso de la enfermedad ${ }^{6}$. La reducción de los niveles de proteínas totales (PT) y albúmina (ALB) son indicativos de daño hepático crónico ${ }^{2,10}$, pero se indica que en la seneciosis, la concentración de proteínas suele ser normal y la albúmina solo disminuiría en las fases finales ${ }^{19}$.

La bilirrubinemia puede mantenerse dentro de los límites normales hasta que se alcanza el estado de insuficiencia funcional; así la bilirrubina total (BRR-T) rara vez supera los $10 \mathrm{mg} / \mathrm{dl}$ y la bilirrubina directa (BRRD) puede representar hasta el $25 \%$. Estos hallazgos coinciden con los encontrados en ambos casos. La urea por lo general es menor a la normal cuando el caballo alcanza el estado de disfunción hepática ${ }^{6}$.

Los APs y sus N-óxidos son un grupo amplio de metabolitos secundarios producidos por ciertas plantas, que pueden resultar hepatotóxicos. Algunos han demostrado tener efectos genotóxicos y carcinogénicos. Los APs no son tóxicos por sí mismos, sino a través de sus productos metabólicos, ya que son transformados por las enzimas hepáticas citocromo P-450 en pirroles, que son los que causan daño a nivel cromosómico ${ }^{29}$.

La molécula de APs comprende un heterociclo pirrolizidínico, con un grupo metilo y un sustituyente hidroxilo (necina). Para que la sustancia sea tóxica, debe presentar una insaturación entre las posiciones 1 y 2 del heterociclo, y una esterificación selectiva del grupo hidroxilo adjunto a la necina. Aislados e identificados en este trabajo, los APs fueron diésteres macrocíclicos de 12 miembros, seis de ellos con base retronecina (1,2-insaturada) a saber: senecionina, integerrimina, usaramina, senecivernina, jacobina y jacozina, con fragmentos típicos a m/z 246, 220, 136 y 120 provenientes de la necina y a 95, 94, 93 debidos a las rupturas del ácido nécico. Los dos restantes, platifilina y neoplatifilina, presentaron fragmentos a m/z 140, 138, 122 y 82 (pico base), característicos de base platinecina 1,2-saturada ${ }^{7}$.

Está descripto que APs con necinas 1,2-insaturadas tales como retronecina, heliotridina y otonecina son tóxicos para los seres humanos y el ganado, especialmente a nivel hepático y pulmonar ${ }^{22}$

La variación en el contenido de APs en las plantas, la cantidad de planta consumida y la susceptibilidad individual de las especies animales, aumentan severamente la intoxicación ${ }^{27}$.

El contenido de APs en la planta varía considerablemente, incrementándose con la maduración de la misma, llegando a su máxima concentración justo en el período anterior a la apertura de los botones florales ${ }^{25}$. Cuando están secas, sufren una pequeña reducción en su contenido de alcaloides, sin embargo se vuelven 
palatables, tornándose riesgosas cuando se incorporan al alimento ${ }^{27}$.

Cabe destacar que en las tres especies de Senecio estudiadas en este trabajo, los APs con base insaturada tóxica, representaronn los compuestos mayoritarios en los extractos alcaloideos, La concentración total de APs del establecimiento El Gallinato osciló entre 8,5 y $19,1 \mathrm{mg} / \mathrm{g}$ (peso seco, material vegetal), mientras que la proveniente de Potrero de Díaz rindió $2,9 \mathrm{mg} / \mathrm{g}$ (peso seco, material vegetal). El Senecio madagascariensis de Gallinato mostró de 3,1 a 7,4 mg/g de peso seco, mientras que la muestra de Potrero de Diaz fue de $6,9 \mathrm{mg} / \mathrm{g}$ (peso seco, material vegetal). Por su parte, $S$. hieronimy de El Gallinato produjo 1,9 a 4,0 mg/g (peso seco, material vegetal).

Estas concentraciones son altas en comparación con la mayoría de otras especies de Senecio tales como S. jacobaea, S. alpinus, S. aquaticus, S. rupestris, $S$. erucifolius, $S$. viscosus, $S$. sylvaticus, $S$. inaequidens y $S$. adonidifolius, cuyos valores oscilan entre $0,30 \mathrm{mg} / \mathrm{g}$ y $2,70 \mathrm{mg} / \mathrm{g}$ (peso seco, material vegetal) ${ }^{15}$.

El diagnóstico de seneciosis y la escasa cantidad de registros de la enfermedad indican que muchas veces estos cuadros pasan desapercibidos. Su naturaleza endémica y las muertes "por goteo" también dificultan el diagnóstico. Todo esto pone en evidencia la necesidad de mantener sistemas de investigación diagnóstica que permitan detectar estos problemas con celeridad. La intoxicación por alcaloides pirrolizidínicos debe ser tenida en cuenta siempre que se observe signología nerviosa en equinos, sobre todo cuando los cuadros presentan características enzoóticas y delimitadas a ciertas regiones.

Considerando el cuadro clínico, los datos bioquímicos, los hallazgos macroscópicos e histopatológicos y la presencia de tres especies de Senecio en las áreas de pastoreo, sumado a la determinación de APs en las mismas, se concluye que los animales afectados sufrieron intoxicación por su ingestión.

Agradecimientos. In memorian, al Ing. Agr. Pablo Saravia, debido a sus contribuciones para el estudio de esta afección a nivel regional. A la Dra. Olga Martinez y al Ing. Lazaro Novara del herbario MCNS de la Facultad de Ciencias Naturales de la Universidad Nacional de Salta por la identificación botánica de las especies. Al Instituto Nacional de Tecnología Agropecuaria, al Consejo de Investigación de la Universidad Católica de Salta y a la Secretaría de Ciencia, Arte e Innovación Tecnológica de la Universidad Nacional de Tucumán, PIUNT 26/D533.

\section{REFERENCIAS}

1. Araya O. 1990. Seneciosis en caballos. Monogr Med Vet Univ Austr Chile 12: 1 (julio).

2. Bain P. 2005. Hígado. En: Patología Clínica Veterinaria (Latimer KS, Mahaffey EA, Prasse KW, ed.), $4^{\circ}$ edición, Multimédica, Barcelona, p. 237-261.
3. Benn M, Gul W. 2007. Pirrolizidine alkaloids in the antipodean genus Brachyglottis (Asteraceae). Biochem Syst Ecol 35: 676-681.

4. Bianchi AR. 2008. Subregiones de la ecorregión noraondina. En: Ecorregión Noroandina. Descripción de subregiones, agroecosistemas, sistemas productivos y cartografia regional (Bianchi AR, Bravo GC ed.), Ediciones INTA, Buenos Aires, p. 19-25.

5. Cabrera AL. 1978. Flora de la Provincia de Jujuy, República Argentina, Senecio, Parte X, Compositae, p. 534-537.

6. Carlson GP. 1999. Ictericia en el caballo. En: Gastroenterología Veterinaria (Anderson NV ed.), $2^{\circ}$ ed., Intermédica, Buenos Aires, p. 630-643.

7. Castells E, Mulder PP, Pérez-Trujillo M. 2014. Diversity of pyrrolizidine alkaloids in native and invasive Senecio pterophorus (Asteraceae): implications for toxicity. Phytochemistry 108: 137-146.

8. Culvenor CC. 1980. Alkaloids and human disease. In: Toxicology in the tropics (Smith RL and Bababunmi EA. Ed.), Taylor \& Francis Ltd, London, p. 124-141.

9. Elcock I, Oehme FW. 1982. Senecio poisoning in horses. A summary. Vet Hum Toxicol 24: 122-123.

10. Frape D. 2013. Equine nutrition and feeding, $4^{\circ}$ ed., Wiley \& Blackwell, Hoboken, New Jersey, 388 p.

11. Ford EJ. 1973. The clinical aspects of ragwort poisoning in horses. Vet Ann 14: 86-88.

12. Gaba A, Barros CS. 1997. Senecio spp poisoning of horses in Southern Brazil. Pesq Vet Bras 17: 36-40.

13. Instituto de Botánica Darwinion. 2013. Flora de la República Argentina. Plantas vasculares. http://www2.darwin. edu.ar/Proyectos/FloraArgentina/Especies.asp?Letra=S.

14. Johnson AE, Molyneux RJ, Ralphs MH. 1989. Senecio: a dangerous plant for man and beast. Rangelands 11: 261264.

15. Macel M, Klinkhamer PG, Vrieling K, Meijden E. 2002. Diversity of pyrrolizidine alkaloids in Senecio species does not affect the specialist herbivore Tyria jacobaeae. Oecologia 133: 541-550.

16. McLean E. 1970. Toxic actions of pyrrolizidine (Senecio) alkaloids. Pharmacol Rev 22: 429.

17. Mattocks AR. 1986. Chemistry and toxicology of pyrrolizidine alkaloids, Academic Press, Orlando (Florida, USA), p. 1-13, 130-157, 158-190.

18. Odriozola E. 2011. Poisoning by plants, mycotoxins and algae in Argentinian livestock. In: Poisoning by plants, mycotoxins and related toxins (Riet-Correa F, Pfister J, Schild AL, Wierenga TL, eds.), Edit. CABI, Oxfordshire.

19. Pearson EG. 2010. Toxicidad por alcaloides pirrolizidínicos. In: Medicina interna de grandes animales (Smith BP ed.), $4^{\circ}$ Ed., Elsevier-Mosby, Barcelona, p. 904-905.

20. Pilati CS. 2007. Intoxicación experimental por Senecio brasiliensis (Asteraseae) em equinos. Pesq Vet Bras 27: 287-452.

21. Rivero R, Matto C, Adrien ML, Alvarez V. 2011. Intoxicación por Senecio spp (Asteraceae) en equinos en Uruguay. Veterinaria (Montevideo) 47: 29-32.

22. Roeder E. 2000. Medicinal plants in China containing pyrrolizidine alkaloids. Pharmazie 55: 711-726. 
23. Rose EF. 1972. Senecio species: toxic plants used as food and medicine in the Transkei. S Afr Med J 46: 1039-1043.

24. Stalker MJ, Hayes MA. 2007. Liver and biliary system In: Pathology of domestic animals (Jubb KV, Kennedy PC, Palmer NC, eds.), $5^{\circ}$ ed., Elsevier, Philadelphia, p. 297-388.

25. Stegelmeier BL. 2011. Pyrrolizidine alkaloid-containing toxic plants (Senecio, Crotalaria, Cynoglossum, Amsinckia, Heliotropium and Echium sp.). Vet Clin North Am - Food Anim Pract 27: 419-428.

26. Summers BA, Cummings JF, Lahunta A. 1995. Veterinary neuropathology, Ed. Mosby, Baltimore, p. 208-211.

27. Tokarnia CH, Farias M, Barbosa JD, Vargas P, Döbereiner J. 2012. Plantas/micotoxinas que afetam o fígado. In: Plantas tóxicas do Brasil, para animais de producción, $2^{\circ}$ ed., Heliantus, Rio de Janeiro.
28. Trigo JR, Leal IR, Matzenbacher NI, Lewinsohn TM. 2003. Chemotaxonomic value of pyrrolizidine alkaloids in southern Brazil Senecio (Senecioneae: Asteraceae). Biochem Syst Ecol 31: 1011-1022.

29. Yaber MA, Ciancia M, Leicach SR. 2009. Variación en la producción de alcaloides en inflorescencias de Senecio grisebachii por deficiencia de nutrientes. Ciencia del suelo (Argentina) 27: 31-39.

\section{SClmago Journal \& Country Rank}

\section{Powered by}

\section{Revista Veterinaria mantiene su índice de impacto}

Noticias de Scimago Research Group (Scimago Journals \& Country Ranks, Scopus-Elsevier) comunican que la publicación de nuestra casa, Revista Veterinaria, aumentó su índice de impacto. El índice SJR mide la influencia científica (impacto) del artículo de una revista, expresando cuán importante es el "artículo promedio" de la publicación en la discusión científica global (sistema Thomson Reuters).

Para nuestra revista, tal indicador había sido de 0,03 entre 2008 y 2011, aumentando a 0,05 en 2012, a 0,11 en 2013, a 0,108 en 2015 y a 0,100 en 2017, último período evaluado. Asimismo, surge para nuestra publicación un sostenido descenso del indicador que relaciona "citas versus autocitas", demostrando que los autores de los artículos están abandonando la práctica de citar sus propias publicaciones anteriores.

Por último, se advierte que según este portal, nuestra "Revista Veterinaria" continúa siendo la única publicación de esta rama de la ciencia que posee índice de impacto en Argentina. Para el resto del cono sur tal distinción recae en Brasil, Chile, Colombia y Venezuela. En revistas de veterinaria, a nivel mundial el mayor índice de impacto (2,45 puntos) recae en Annual Review of Animal Biosciences (Estados Unidos). 